\title{
Detección temprana de la violencia del compañero íntimo en el sector sanitario. ¿Una intervención basada en la evidencia?
}

\author{
Carmen Vives-Cases ${ }^{\mathrm{a}, \mathrm{b}}$, Diana Gil-González ${ }^{\mathrm{a}, \mathrm{c}}$, Mercedes Carrasco-Portiño \\ y Carlos Álvarez-Dardeta,b,c
}

${ }^{a}$ Área de Medicina Preventiva y Salud Pública. Universidad de Alicante. Alicante.

${ }^{\mathrm{b}}$ Red Nacional de Investigación sobre Salud y Género.

'Observatorio de Políticas Públicas y Salud (OPPS). Universidad de Alicante. Alicante. España.

El sector sanitario se muestra como un claro instrumento en la lucha contra la violencia del compañero íntimo (VCI). Las propias consecuencias de este problema para la salud dan a los sanitarios la responsabilidad y la oportunidad de participar en las intervenciones de $\mathrm{VCl}^{1-3}$. Las instituciones políticas, conscientes de esta realidad, cuentan con el sector sanitario para el desarrollo de sus respuestas al problema ${ }^{4,5}$. Así, en la Ley Orgánica de Medidas de Protección Integral contra la Violencia de Género ${ }^{6}$, se atribuye a las administraciones sanitarias la responsabilidad de promover medidas para la identificación temprana de mujeres afectadas. Se trata de una iniciativa recomendada por la Organización Mundial de la Salud ${ }^{7}$ y la Sociedad Española de Medicina de Familia y Comunitaria ${ }^{8}$.

La ampliación de responsabilidades del sector sanitario, entre otros sectores, manifestada en la Ley de Protección Integral contra la Violencia de Género es reflejo del creciente compromiso adquirido por parte de las instituciones políticas respecto a la $\mathrm{VCl}$. La detección temprana de maltratos podría considerarse un medio para fortalecer la prevención secundaria del problema ${ }^{9}$. Sin embargo, la VCl en España es una epidemia que no parece haber disminuido a pesar de las medidas hasta el momento adoptadas ${ }^{10-12}$. Por este motivo, y dada la importancia de contribuir al desarrollo de políticas basadas en la evidencia, la propuesta de promover la detección temprana del maltrato en los servicios sanitarios españoles merece cierta reflexión.

El objetivo de este estudio es determinar si la realización de una prueba para la detección temprana de la VCl a todas las mujeres que acudan a los servicios sanitarios es adecuada en términos de efectividad, aceptabilidad y reducción del riesgo de recurrencias ${ }^{13}$, así como realizar una estimación de los posibles resultados del cribado extrapolando la información disponible sobre el tema a la población de mujeres españolas.

\section{Material y método}

Se ha llevado a cabo una búsqueda bibliográfica en 2 etapas. La primera, del 26 de enero al 2 de febrero de 2005, en las bases de datos de MEDLINE e ISI Web of Knowledge, se limitó a estudios con resúmenes disponibles publicados entre 2000 y 2005. El criterio de inclusión de los artículos fue la recuperación de revisiones sistemáticas que se plantearan, entre sus principales objetivos, evaluar la efectividad de los protocolos para la detección temprana del maltrato en mujeres que acuden a los centros de atención primaria, urgencias $u$ hospitalaria, determinar el grado de aceptabilidad de este tipo de intervenciones en profesionales sanitarios y mujeres afectadas, y/o analizar la capacidad de la medida para reducir el riesgo de recurrencias y mejorar la calidad de vida de la mujeres afectadas.

La segunda etapa de la búsqueda bibliográfica se realizó entre el 6 y el 19 de abril de 2005 en las siguientes bases de datos: MEDLINE, CINAHL, Psycoin-

Correspondencia: Dra. C. Vives-Cases.

Departamento de Salud Pública. Edificio de Ciencias Sociales.

Campus Sant Vicent de Raspeig. Universidad de Alicante.

Apdo. 99. 03080 Alicante. España.

Correo electrónico: carmen.vives@ua.es fo (enero de 2003 a marzo de 2005), ISI Web of Knowledge -Web of Science, Current Contents Connect y ISI Proceedings- (1945-2005), DARE (todos los años), HSTAT (todos los años), Cochrane Plus (1800-2005), SUM Search (1994-2005), ACP Journal Club (1996-2005), Evidence-Based Medicine (2000-2005), Clinical Evidence (todos los años), Bandolier (1998-2005), Tripdatabase (1997-2005), BestBets (1994-2005), LILACS (1965-2005), National Guideline Clearinghouse (2000-2005) y BDENF (1965-2005). El criterio de inclusión fue revisiones sistemáticas o artículos originales que plantearan, entre sus principales objetivos, los anteriormente mencionados.

En ambas etapas se excluyeron los estudios transversales basados en la población general, investigaciones sobre la etiología de la VCl, artículos sobre otros aspectos relacionados con la aplicación del test, otros tipos de violencia, otras víctimas u otros tipos de intervenciones, revisiones teóricas, editoriales y referencias sin resumen disponible.

Las palabras clave utilizadas fueron "screening and battered women" 0 "screening and violence against women" o «screening and domestic violence», identificadas en las bases datos que contaban con descriptores o vocabulario controlado (tesauros) -MEDLINE, Cinahl y Psycoinfo-. En las bases de datos que no cuentan con esta herramienta se utilizaron las mismas palabras clave. En el presente artículo se describen los objetivos, el diseño, el ámbito de actuación, el tamaño de la muestra, los resultados y las conclusiones de los estudios finalmente seleccionados. También se realiza una estimación sumaria de la especificidad, la sensibilidad, los valores predictivos positivo y negativo de las pruebas de detección temprana del maltrato evaluadas. Para ello, se calculó la media de las puntuaciones ofrecidas en los estudios sobre estos indicadores. A partir de los resultados obtenidos, se calculó el número de falsos positivos y negativos que se darían teniendo en cuenta la prevalencia de mujeres maltratadas en España según la macroencuesta del Instituto de la Mujer de $2002^{14}$ y el número de mujeres españolas en 2004 según el Instituto Nacional de Estadística15.

\section{Resultados}

Se localizaron 897 estudios, de los que se rechazaron 879 , sobre todo porque tratan tipos de violencias y víctimas diferentes de la $\mathrm{VCl}$ u otro tipo de intervenciones (tabla 1 ). Entre los 18 finalmente incluidos para el análisis, se recuperaron 5 revisiones sistemáticas ${ }^{16-20}$ y 13 estudios originales $^{21-33}$. Tres de estos 13 originales ya se habían analizado en las revisiones sistemáticas recuperadas ${ }^{21-23}$. El estudio con información más actualizada es una revisión sistemática publicada en $2005^{20}$ (tabla 2).

\section{TABLA 1}

Artículos excluidos en la revisión sobre protocolos de detección temprana de violencia del compañero íntimo ( $\mathrm{VCl})$ contra mujeres

\begin{tabular}{|l|c|}
\hline \multicolumn{1}{|c|}{ Criterios de exclusión } & Número (\%) \\
\hline Artículos duplicados entre todas las bases de datos & 264 \\
Estudios sobre otros tipos de violencia y otros tipos & \\
de víctimas & $178(20,25)$ \\
Evaluaciones de otros tipos de intervenciones & $155(17,63)$ \\
sobre el problema & $142(16,15)$ \\
Estudios transversales basados en la población general & $58(6,60)$ \\
Estudios de otros aspectos del cribado de VCl & $38(4,32)$ \\
Investigaciones sobre la etiología de la VCl & $38(4,32)$ \\
Artículos sin resumen & $5(0,57)$ \\
Revisiones teóricas sobre VCl y cribado de maltratos & $1(0,11)$ \\
Editoriales & $879(100)$ \\
Total & \\
\hline
\end{tabular}


TABLA 2

Características de los estudios sobre la efectividad, aceptabilidad y consecuencias de la detección temprana de la violencia del compañero íntimo (VCl) contra las mujeres

\begin{tabular}{|c|c|c|c|c|c|c|c|}
\hline Referencia & Año & País & Objetivo & Diseño & Ámbito & $\begin{array}{c}\text { Tamaño de la } \\
\text { muestra }\end{array}$ & Principal resultado \\
\hline 16 & 2002 & Gran Bretaña & $\begin{array}{l}\text { Analizar la aceptabilidad } \\
\text { y la efectividad de una } \\
\text { prueba de detección } \\
\text { temprana del maltrato } \\
\text { en mujeres }\end{array}$ & $\begin{array}{l}\text { Revisión } \\
\text { sistemática }\end{array}$ & $\begin{array}{l}\text { Servicios } \\
\quad \text { sanitarios } \\
\quad \text { (en general) }\end{array}$ & 20 artículos & $\begin{array}{l}\text { La aplicación de programas } \\
\text { de detección temprana de } \\
\text { la VCI no está justificada }\end{array}$ \\
\hline 17 & 2004 & EE.UU. & $\begin{array}{l}\text { Examinar la evidencia } \\
\text { de los procedimientos } \\
\text { y las intervenciones } \\
\text { de detección temprana }\end{array}$ & $\begin{array}{l}\text { Revisión } \\
\text { sistemática }\end{array}$ & $\begin{array}{l}\text { Atención } \\
\text { primaria }\end{array}$ & $\begin{array}{l}0 \text { artículos } \\
\text { sobre la VCI } \\
\text { contra } \\
\text { mujeres }\end{array}$ & $\begin{array}{l}\text { No hay información sobre } \\
\text { las consecuencias de las } \\
\text { intervenciones de } \\
\text { detección temprana de } \\
\text { mujeres víctimas de } \mathrm{VCl}\end{array}$ \\
\hline 18 & 2004 & EE.UU. & $\begin{array}{l}\text { Examinar la evidencia de los } \\
\text { daños y beneficios de la } \\
\text { detección temprana del } \\
\text { maltrato en mujeres }\end{array}$ & $\begin{array}{l}\text { Revisión } \\
\text { sistemática }\end{array}$ & $\begin{array}{l}\text { Servicios } \\
\text { sanitarios (en } \\
\text { general) }\end{array}$ & $\begin{array}{l}14 \text { artículos } \\
\quad \text { sobre la VCI } \\
\text { contra } \\
\text { mujeres }\end{array}$ & $\begin{array}{l}\text { Escasos estudios proveen } \\
\text { datos sobre la detección } \\
\text { y manejo del cribado }\end{array}$ \\
\hline 19 & 2003 & EE.UU. & $\begin{array}{l}\text { Revisar sistemáticamente } \\
\text { los estudios sobre } \\
\text { detección temprana } \\
\text { de la VCl para identificar } \\
\text { víctimas y reducir la } \\
\text { morbilidad y la mortalidad } \\
\text { por esta causa }\end{array}$ & $\begin{array}{l}\text { Revisión } \\
\text { sistemática }\end{array}$ & $\begin{array}{l}\text { Servicios de } \\
\text { urgencias }\end{array}$ & 17 artículos & $\begin{array}{c}\text { No hay suficiente evidencia } \\
\text { ni a favor ni en contra de } \\
\text { la aplicación del cribado } \\
\text { de la VCI en urgencias }\end{array}$ \\
\hline 20 & 2004 & EE.UU. & $\begin{array}{l}\text { Evaluar los daños } \\
\text { y beneficios del cribado } \\
\text { de la } \mathrm{VCl}\end{array}$ & $\begin{array}{l}\text { Revisión } \\
\text { sistemática }\end{array}$ & $\begin{array}{l}\text { Especialidad } \\
\text { en lesiones } \\
\text { dentales } \\
\text { o faciales }\end{array}$ & 0 artículos & $\begin{array}{l}\text { No hay ensayos } \\
\text { aleatorizados controlados } \\
\text { que hagan pertinente la } \\
\text { aplicación del cribado de }\end{array}$ \\
\hline 24 & 1996 & EE.UU. & $\begin{array}{l}\text { Describir el desarrollo, } \\
\text { el diseño y la validez } \\
\text { de una prueba de } \\
\text { detección temprana } \\
\text { de la VCl }\end{array}$ & Ensayo clínico & $\begin{array}{l}\text { Servicio de } \\
\text { urgencias }\end{array}$ & 595 mujeres & $\begin{array}{l}\text { la VCI } \\
\text { La prueba obtuvo una } \\
\text { sensibilidad del 50\% } \\
\text { y una especificidad del } \\
\text { 95\%. Su aplicación no } \\
\text { es recomendable }\end{array}$ \\
\hline 25 & 2003 & Canadá & $\begin{array}{l}\text { Evaluar la validez de } 3 \text { tipos } \\
\text { de test para la detección } \\
\text { temprana de la VCl }\end{array}$ & $\begin{array}{l}\text { Cohortes } \\
\text { retrospectivo }\end{array}$ & Hospital & 75 mujeres & $\begin{array}{l}\text { Cada prueba obtiene } \\
\text { resultados diferentes. } \\
\text { En la primera, una } \\
\text { sensibilidad del } 96 \% \text { y una } \\
\text { especificidad del } 75 \% \text {; en la } \\
\text { segunda, una sensibilidad } \\
\text { del } 89 \% \text { y una especificidad } \\
\text { del } 100 \% \text {, y en la tercera, } \\
\text { una sensibilidad del } 83 \% \\
\text { y una especificidad del } \\
100 \% \text {. La sensibilidad } \\
\text { global es del } 83 \% \text { y la } \\
\text { especificidad del } 91,6 \%\end{array}$ \\
\hline 26 & 2004 & EE.UU. & $\begin{array}{l}\text { Determinar si un breve } \\
\text { cribado predice futuras } \\
\text { situaciones de la } \mathrm{VCl}\end{array}$ & $\begin{array}{l}\text { Cohortes } \\
\text { prospectivo }\end{array}$ & $\begin{array}{l}\text { Servicio de } \\
\text { urgencias }\end{array}$ & 215 mujeres & $\begin{array}{l}\text { El cribado identifica a } \\
\text { mujeres con riesgo alto } \\
\text { de VCl física y verbal }\end{array}$ \\
\hline 27 & 2003 & EE.UU. & $\begin{array}{c}\text { Analizar la validez de una } \\
\text { pregunta para detectar } \\
\text { tempranamente la } \mathrm{VCl}\end{array}$ & $\begin{array}{l}\text { Encuesta } \\
\text { transversal }\end{array}$ & $\begin{array}{l}\text { Atención } \\
\text { primaria }\end{array}$ & 399 mujeres & $\begin{array}{l}\text { Sensibilidad del } 8,8 \% \text { y } \\
\text { especificidad del } 91,2 \% \text {. } \\
\text { Los resultados obtenidos } \\
\text { cuestionan la utilidad de } \\
\text { la pregunta }\end{array}$ \\
\hline 28 & 2002 & EE.UU. & $\begin{array}{l}\text { Determinar si un cribado } \\
\text { de la VCl incrementa } \\
\text { el conocimiento sobre } \\
\text { el tema }\end{array}$ & $\begin{array}{l}\text { Encuesta } \\
\text { transversal }\end{array}$ & $\begin{array}{l}\text { Atención } \\
\text { primaria }\end{array}$ & 1.402 mujeres & $\begin{array}{l}\text { El cribado incrementa el } \\
\text { conocimiento sobre la } \\
\text { prevalencia de la } \mathrm{VCl}\end{array}$ \\
\hline 29 & 2002 & Italia & $\begin{array}{l}\text { Evaluar la factibilidad } \\
\text { de preguntar a las mujeres } \\
\text { si sufren mediante la } \mathrm{VCl}\end{array}$ & $\begin{array}{l}\text { Encuesta } \\
\text { transversal }\end{array}$ & $\begin{array}{l}\text { Servicios } \\
\quad \text { sanitarios (en } \\
\text { general) }\end{array}$ & 510 mujeres & $\begin{array}{l}\text { Los profesionales tienen que } \\
\text { ser formados para hacer } \\
\text { factible la medida }\end{array}$ \\
\hline 30 & 2002 & Francia & $\begin{array}{l}\text { Medir la prevalencia de } \\
\text { la } \mathrm{VCI} \text { mediante un } \\
\text { protocolo para la } \\
\text { detección temprana }\end{array}$ & $\begin{array}{l}\text { Encuesta } \\
\text { transversal }\end{array}$ & $\begin{array}{l}\text { Servicio de } \\
\text { urgencias }\end{array}$ & 126 mujeres & $\begin{array}{l}\text { Prevalencia detectada } \\
\quad \text { del } 18 \%\end{array}$ \\
\hline 31 & 2003 & EE.UU. & $\begin{array}{l}\text { Analizar el grado de } \\
\text { aplicación de un } \\
\text { protocolo de detección } \\
\text { temprana de la VCl } \\
\text { entre los clínicos }\end{array}$ & Serie de casos & $\begin{array}{r}\text { Servicios de } \\
\text { urgencias }\end{array}$ & 527 casos & $\begin{array}{l}\text { La aplicación del cribado } \\
\text { está lejos de alcanzar un } \\
\text { grado óptimo }\end{array}$ \\
\hline 32 & 2004 & EE.UU. & $\begin{array}{l}\text { Analizar las actitudes } \\
\text { de los médicos residentes } \\
\text { sobre cribado de la VCl }\end{array}$ & $\begin{array}{l}\text { Encuesta } \\
\text { transversal }\end{array}$ & $\begin{array}{l}\text { Hospitales } \\
\text { universitarios }\end{array}$ & $\begin{array}{l}103 \text { residentes } \\
\text { de medicina } \\
\text { interna }\end{array}$ & $\begin{array}{l}\text { Muchos residentes están a } \\
\text { favor de la iniciativa. } \\
\text { Opinan que su aplicación } \\
\text { sería más efectiva con una } \\
\text { previa formación y } \\
\text { sensibilización en el tema }\end{array}$ \\
\hline 33 & 2002 & Gran Bretaña & $\begin{array}{l}\text { Analizar el grado de } \\
\text { aceptabilidad del cribado } \\
\text { entre mujeres y } \\
\text { profesionales sanitarios }\end{array}$ & $\begin{array}{l}\text { Encuesta } \\
\text { transversal }\end{array}$ & $\begin{array}{l}\text { Servicios } \\
\text { sanitarios (en } \\
\text { general) }\end{array}$ & $\begin{array}{l}1.207 \text { mujeres, } \\
107 \\
\text { profesionales } \\
\text { sanitarios }\end{array}$ & $\begin{array}{l}\text { Los resultados no hacen } \\
\text { convincente la aplicación } \\
\text { de protocolos para la } \\
\text { detección temprana de la } \\
\text { VCl }\end{array}$ \\
\hline
\end{tabular}




\section{Efectividad de la prueba diagnóstica}

No hay ensayos aleatorizados diseñados para medir la efectividad de pruebas ya utilizadas para la detección temprana de la $\mathrm{VCl}^{18-20}$. La heterogeneidad de las variables y los instrumentos de referencia que utilizan dificulta la comparación de sus resultados ${ }^{17}$.

A partir de los resultados disponibles sobre la exactitud de las pruebas de detección temprana aplicadas ${ }^{24,25,27}$ (tabla 2) se obtuvo una sensibilidad sumaria del 47,2\%, una especificidad del $92,6 \%$, un valor predictivo positivo del $44 \%$ y negativo del $93,4 \%$. Teniendo en cuenta que la prevalencia de mujeres maltratadas en España es del 11,1\% ( $n=2.432 .279$ sobre población de mujeres españolas en 2004 de 21.912.433), se estima que 160.530 mujeres serían erróneamente etiquetadas como maltratadas y 1.362 .076 casos reales no serían detectados por la prueba de detección temprana (tabla 3).

\section{Grado de aceptación de la intervención}

Según resultados de una revisión sistemática, la proporción de mujeres a favor de la realización de pruebas para la detección temprana de la VCI oscila entre un 43 y un $85 \%$ de las entrevistadas, mientras que la proporción de médicos a favor es de un $33 \% 16$

En artículos originales, se estima un porcentaje de aceptación del $80 \%$ en el caso de las mujeres ${ }^{33}$ y del $63 \%$ en el de los residentes de varios hospitales universitarios ${ }^{32}$.

\section{Riesgo de recurrencias y mortalidad por violencia del compañero íntimo}

No hay estudios que ofrezcan información sobre las consecuencias de la detección temprana del maltrato en mujeres en cuanto a la reducción del riesgo de recurrencias y mejora de la calidad de vida de las mujeres afectadas ${ }^{16-20,32}$.

\section{Discusión}

No hay suficiente evidencia para afirmar que las mujeres se benefician clínicamente de las pruebas de detección temprana del maltrato. Es urgente la realización de ensayos aleatorizados controlados sobre esta cuestión. La aplicación masiva de pruebas de detección temprana de la $\mathrm{VCl}$ en España proporcionaría resultados poco útiles. La aceptación de la medida por parte de las mujeres y los profesionales sanitarios es necesaria para legitimar su aplicación.

La estimación de los posibles resultados de la prueba de detección temprana de la VCI en España es limitada por razones metodológicas y por la heterogeneidad de la información utilizada para su cálculo. Sin embargo, ofrece una información útil sobre la efectividad esperada de la aplicación de una prueba de detección temprana del maltrato similar a las existentes a todas las mujeres que acuden a los servicios sanitarios españoles.

La información disponible, en principio, no justifica la pertinencia de aplicar masivamente pruebas para la detección temprana de la $\mathrm{VCl}$ en los servicios sanitarios españoles ${ }^{15}$. Sin embargo, esto no exime a las mujeres afectadas de su derecho a una atención sociosanitaria de calidad. Los profesionales sanitarios tienen la responsabilidad de ofrecer e informar de los recursos existentes para abordar la VCl. También deben fortalecer sus habilidades para el reconocimiento de signos y síntomas de maltrato ${ }^{8}$.

La aplicación de una prueba de detección temprana del maltrato en los servicios sanitarios españoles precisa un previo esfuerzo por incrementar la evidencia empírica sobre el tema. Se necesita al menos un ensayo aleatorizado y con-
TABLA 3

Estimación sumaria de la sensibilidad, la especificidad, los valores predictivos y los falsos positivos y negativos de la prueba de detección temprana de la violencia del compañero íntimo en una hipotética aplicación en los servicios sanitarios españoles

\begin{tabular}{|l|c|c|c|}
\hline Resultados de la prueba & $\begin{array}{c}\text { Mujeres } \\
\text { maltratadas }\end{array}$ & $\begin{array}{c}\text { Mujeres } \\
\text { no maltratadas }\end{array}$ & Total \\
\hline Positivo & 1.070 .203 & 160.530 & 1.230 .733 \\
Negativo & 1.362 .076 & 19.319 .624 & 20.681 .700 \\
Total & 2.432 .279 & 19.480 .154 & 21.912 .433 \\
\hline
\end{tabular}

Estimación sumaria de las pruebas: sensibilidad, 0,472; especificidad, 0,926. Prevalencia de mujeres maltratadas en España: $11,1 \%$. Valor predictivo positivo: 0,44 . Valor predictivo negativo: 0,934

trolado que permita conocer la efectividad de la prueba y facilite la toma de decisiones políticas basadas en la evidencia. El objetivo de la evaluación también debería incluirse entre las responsabilidades de las administraciones sanitarias si se pretende obtener una mejora real del problema. De lo contrario, la aplicación de pruebas para la detección temprana sería otro ejemplo de intervenciones de $\mathrm{VCl}$ en que no se tiene previsto un sistema de evaluación ${ }^{34-37}$

La aplicación del instrumento no parece estar garantizada, sobre todo por la actitud mostrada por los profesionales médico-sanitarios. En la Ley Integral de Violencia de Género está prevista la realización de cursos de formación y sensibilización para promover la participación de los profesionales $^{8}$. Además, las actitudes pueden haber cambiado por la creciente concienciación social sobre el problema y el hecho de que la detección temprana de la VCl se haya formalizado en la misma ley ${ }^{32}$.

En la bibliografía también se ha recogido cierto miedo por parte de los profesionales a los efectos adversos de la detección temprana del maltrato ${ }^{38}$. La estimación realizada no permite descartar esta posibilidad y apunta a que la aplicación de la prueba a todas las mujeres que acuden a los servicios sanitarios españoles podría generar daños como el hecho de ser etiquetada como una maltratada cuando en realidad no es así3 ${ }^{39}$. Además, no parece que este perjuicio quedara compensado con la efectividad de la prueba. Esclarecer estos resultados no sólo es útil para los sanitarios, sino que tiene también un componente ético.

Incrementar la evidencia y asegurar la efectividad, la aceptabilidad y la utilidad de las pruebas de detección temprana de la VCl no sólo es parte de la calidad de la atención a la que tienen derecho las mujeres afectadas, sino que también puede ser un punto de partida clave para la generación de otros tipos de intervenciones sobre el problema realizadas desde el sistema sanitario.

\section{REFERENCIAS BIBLIOGRÁFICAS}

1. Velzeboer M, editor. Violence against women: the health sector responds. Washington DC: PAHO; 2003

2. Krug E, Dahlberg L, Mercy J, Zwi A, Lozano R, editores. Informe mundial sobre la violencia y la salud. Washington: Organización Panamericana de la Salud, Oficina Sanitaria Panamericana, Oficina Regional de la Organización Mundial de la Salud; 2002. Informe técnico n. 588.

3. Plazaola-Castaño J, Ruiz-Pérez I. Violencia contra la mujer en la pareja y consecuencias en la salud física y psíquica. Med Clin (Barc). 2004;122: 461-7.

4. Ministerio de Trabajo y Asuntos Sociales. Plan de Acción contra la violencia doméstica 1998-2000. Madrid: Instituto de la Mujer; 1998.

5. Ministerio de Trabajo y Asuntos Sociales. II Plan Integral contra la violencia doméstica 2001-2004. Revista del Ministerio de Trabajo y Asuntos Sociales. 2001;40:125-34

6. Ley Orgánica de Medidas de Protección Integral contra la Violencia de Género. Boletín General de las Cortes Generales. 2004,21760;195-232. 
7. Women's Health and Development. Violence against women: a priority health issue. Geneva: World Health Organization; 1997. p. 27-30 [consultado 18/04/2005]. Versión en español disponible en: http://www.who. int/gender/violence/en/violencia_infopack1.pdf

8. Fernández MC, Herrero S, Buitrago F, Ciurana R, Chocron L, García J, et al. Violencia doméstica. Madrid: Sociedad Española de Medicina de Familia y Comunitaria, Ministerio de Sanidad y Consumo; 2003.

9. Last J. Public health and human ecology. East Norfolk: Appleton and Lange; 1987.

10. Vives C, Álvarez-Dardet C, Caballero P. Violencia del compañero íntimo en España. Gac Sanit. 2003;17:268-74.

11. Vives-Cases C, Caballero P, Álvarez-Dardet C. Análisis temporal de la violencia del compañero íntimo. Gac Sanit. 2004;18:346-50.

12. Vives-Cases C, Álvarez-Dardet C, Colomer C, Bernabeu MA. Una experiencia de Defensa de la salud sobre violencia de género. Gac Sanit. 2005; 19:262-4.

13. Miller A, Goel V. Screening. En: Detels R, McEwen J, Beaglehole R, Tanaka $H$, editors. Oxford textbook of public health. The practice of public Health. 4th ed. Oxford: Oxford University Press; 2002. p. 1823-37.

14. Instituto de la Mujer. Macroencuesta de violencia contra las mujeres. Porcentaje de mujeres maltratadas según comunidades autónomas. Madrid: Instituto de la Mujer; 2004 [consultado 26/04/2005]. Disponible en: http://www.mtas.es/mujer/mcifras/principa2.htm

15. Población de mujeres españolas a 1 de enero de 2004. Demografía y población. Instituto Nacional de Estadística [consultado 27/04/2005]. Disponible en: http://www.ine.es/inebase/cgi/axi

16. Ramsay J, Richardson J, Carter YH, Davidson LL, Feder G. Should health professionals screen women for domestic violence? Systematic Review. BMJ. 2002;325:314-27.

17. Nelson $H$, Nygren $P$, Mclnerney $Y$. Screening for family and intimate partner violence. Rockville: Agency for Health Care Research and Quality (AHRQ); 2004.

18. Nelson $H$, Nygren $P$, Mclnerney $Y$, Klein J. Screening women and elderly adults for family and intimate partner violence: a review of the evidence for the USA Preventive Services Task Force. Ann Intern Med. 2004:140:387-96.

19. Anglin D, Sachs C. Preventive care in the emergency department: screening for the domestic violence in the emergency department. Acad Emerg Med. 2003;10:1118-27.

20. Coulthard P, Yong S, Adamson L, Warburton A, Worthington HV, Esposito $M$. Cribaje (screening) sobre violencia doméstica y programas de intervención para adultos con lesiones dentales o faciales (revisión Cochrane traducida). En: La biblioteca Cochrane Plus, 2005; n. ${ }^{\circ} 1$. Oxford: Update Software Ltd. Disponible en: http://www.update-software.com

21. Koziol-McLain J, Coates CJ, Lowenstein SR. Predictive validity of a screen for partner violence against women. Am J Prev Med. 2001;21:93-100.

22. McCaw B, Berman W, Syme L, Hunkeler EF. Beyond screening for domestic violence. A systems model approach in a managed care setting. Am J Prev Med. 2001;21:170-6.
23. Feldhaus KM, Koziol McLain J, Amsbury HL, Norton IM, Lowenstein SR Abott JT. Accuracy of 3 brief questions for detecting partner violence in the emergency department. JAMA. 1997;277:1357-61.

24. Waller AE, Hohenhaus SM, Shah PJ, Stern EA. Development and validation of an emergency department screening. An referral protocol for victims of domestic violence. Ann Emerg Med. 1996;27:754-60.

25. Paranjape A, Liebschutz J. A three-question screen for intimate partner violence. J Women's Health Gend Based Med. 2003;12:233-9.

26. Houry D, Feldhaus K, Peery B, Abbott J, Lowenstein SR, Al-Baata-deMontero S, et al. A positive domestic violence screen predicts future domestic violence. J Interpers Violence. 2004:19:955-66.

27. Peralta RL, Fleming MC. Screening for intimate partner biolence in a primary care setting. The validity of «feeling safe at home» and prevalence results. J Am Board Fam Pract. 2003;16:525-32.

28. Coker AL, Bethea L, Smith PH, Fadden MK, Brandt M. Missed opportunities: intimate partner violence in family practice settings. Prev Med. 2002;34:445-54

29. Romito P, Gerin D. Asking patients about violence: a survey of 510 women attending social and health services in Trieste, Italy. Soc Sci Med. 2002;54:1813-24.

30. Lejoyeux M, Zilhardt $P$, Chièze $F$, Fichelle $A$, Loughlin M, Poujade $A$, et al. Screening for domestic violence among patients admitted to a French emergency service. Eur Psychiatry. 2002;17:479-83.

31. Richter KP, Surprenant ZJ, Schmelzle KH, Mayo MS. Detecting and documenting intimate partner violence -An intake for question is not enough. Violence Against Women. 2003:9:458-65.

32. Varjavand N, Cohen DG, Gracely EJ, Novack DH. A Survey of residents' attitudes and practices in screening for, managing, and documentating domestic violence. JAMA. 2004;59:48-53.

33. Richardson J, Coid J, Petruckevitch A Shan Chung W Moorey S, Feder G. Identifying domestic violence: cross sectional study in primary care. BMJ. 2002;324:1-6.

34. Buzawa ES, Buzawa CG, editors. Do arrest and restraining orders work? Thousand Oaks: Sage; 1996. p. 43-53, 115-49.

35. Davis L, Hagen J, Early T. Social services for battered women: are they adequate, accessible, and appropiate? Soc Work. 1996;39:695-704.

36. Sullivan C, Basta J, Tan C, Davidson WS. Afther the crisis: a needs assessment of women leaving a domestic violence shelter. Violence Vict. 1992; 7:267-75.

37. Tutty L. Post-shelter services: the efficacy of follow-up programs for abused women. Res Soc Work Pract. 1996;6:425-41.

38. Waalen J, Goodwin M, Spitz A, Petersen R, Saltzman L. Screening for Intimate Partner Violence by Health Care Providers. Barriers and Interventions. Am J Prev Med. 2000;19:230-7.

39. Thurston WE, Cory J, Scout C. Building a feminist theoretical framework for screening of wife battering: key issues to be assessed. Patient Educ Couns. 1998;33:299-304. 